\title{
Food portion sizes and their relationship with intake and quality of fat in Irish children
}

\author{
J. Lyons, J. Walton and A. Flynn \\ School of Food and Nutritional Sciences, University College Cork, Republic of Ireland
}

It is now widely accepted that food portion sizes may influence energy intake. However, data are sparse on the relationship between food portion size and markers of dietary quality in free-living populations. Data from the Irish National Children's Food Survey (NCFS) were used to investigate associations between portion sizes of selected foods and intake and quality of fat in Irish children. The NCFS was carried out between 2003 and 2004 to establish a database of habitual food and drink consumption in a representative sample of Irish children ( $n$ 594) aged 5-12 years. Intake data were collected using a 7-d weighed food record. Analysis of nutrients was carried out using WISPC (Tinuviel Software, Anglesey, UK), which contains data from McCance and Widdowson's The Composition of Foods, 6th edition $^{(1)}$.

Portion size $(\mathrm{g})$, defined as the weight of food consumed per eating occasion, was estimated for each day the food was consumed. Where a food was consumed on more than one occasion on $1 \mathrm{~d}$, the largest portion size was assigned. The portion size data were split by tertile, stratified by gender and age group as appropriate. Intakes of fat and saturated fat as \% total energy (TE) (mean values) were examined across tertiles for the days on which the foods were consumed.

\begin{tabular}{|c|c|c|c|c|c|c|c|c|c|}
\hline & \multicolumn{4}{|c|}{ Total fat as $\% \mathrm{TE}$} & \multicolumn{4}{|c|}{ Saturated fat as $\% \mathrm{TE}$} & \multirow[b]{2}{*}{ Tertile medians $(\mathrm{g})$} \\
\hline & $\mathrm{T} 1$ & T1 & T3 & $\overline{\mathrm{P}}$ & $\mathrm{T} 1$ & $\mathrm{~T} 2$ & T3 & $\mathrm{P}[\mathrm{T} 1, \mathrm{~T} 2, \mathrm{~T} 3]$ & \\
\hline Pizza & 33.1 & 34.2 & 33.9 & & 13.5 & 14.4 & 14.4 & & $66,107,219$ \\
\hline White bread and rolls & $34.1^{\mathrm{a}}$ & $33.4^{\mathrm{a}}$ & $32.4^{\mathrm{b}}$ & $\downarrow$ & $14.4^{\mathrm{a}}$ & $14.0^{\mathrm{a}}$ & $13.3^{\mathrm{b}}$ & $\downarrow$ & $37,66,84$ \\
\hline Wholemeal/brown bread and rolls & $34.4^{\mathrm{a}}$ & $33.7^{\mathrm{ab}}$ & $32.7^{\mathrm{b}}$ & $\downarrow$ & $14.4^{\mathrm{a}}$ & $13.7^{\mathrm{ab}}$ & $13.3^{\mathrm{b}}$ & $\downarrow$ & $33,60,88$ \\
\hline Potatoes, boiled & $34.2^{\mathrm{a}}$ & $32.8^{\mathrm{b}}$ & $31.5^{\mathrm{c}}$ & $\downarrow$ & $14.7^{\mathrm{a}}$ & $14.1^{\mathrm{a}}$ & $13.2^{\mathrm{b}}$ & $\downarrow$ & $66,129,212$ \\
\hline Chips & 34.5 & 35.0 & 35.1 & & 14.0 & 13.9 & 13.4 & & $61,102,171$ \\
\hline Ready-to-eat breakfast cereals & $33.6^{\mathrm{a}}$ & $32.7^{\mathrm{b}}$ & $31.5^{\mathrm{c}}$ & $\downarrow$ & $14.2^{\mathrm{a}}$ & $14.0^{\mathrm{a}}$ & $13.5^{\mathrm{b}}$ & $\downarrow$ & $21,37,57$ \\
\hline Fruit, excluding dried fruit & $33.5^{\mathrm{a}}$ & $32.6^{\mathrm{a}}$ & $31.7^{\mathrm{b}}$ & $\downarrow$ & $14.4^{\mathrm{a}}$ & $13.7^{\mathrm{b}}$ & $13.2^{\mathrm{b}}$ & $\downarrow$ & $65,115,162$ \\
\hline Vegetables, excluding pulses & $34.0^{\mathrm{a}}$ & $33.3^{\mathrm{ab}}$ & $32.9^{\mathrm{b}}$ & $\downarrow$ & $14.5^{\mathrm{a}}$ & $13.8^{\mathrm{b}}$ & $13.8^{\mathrm{b}}$ & $\downarrow$ & $22,50,90$ \\
\hline Roast meats & 32.8 & 32.8 & 32.5 & & 14.1 & 13.8 & 13.6 & & $27,53,100$ \\
\hline Frying meats & $35.1^{\mathrm{a}}$ & $37.1^{\mathrm{b}}$ & $38.7^{\mathrm{c}}$ & $\uparrow$ & 14.7 & 14.8 & 15.0 & & $27,47,75$ \\
\hline Eggs, boiled/fried/scrambled & $36.7^{\mathrm{a}}$ & $36.7^{\mathrm{ab}}$ & $38.7^{\mathrm{b}}$ & $\uparrow$ & $15.0^{\mathrm{a}}$ & $14.2^{\mathrm{ab}}$ & $15.9^{\mathrm{ac}}$ & $\uparrow$ & $43,60,100$ \\
\hline Milk, all types & $32.7^{\mathrm{a}}$ & $33.6^{\mathrm{b}}$ & $34.1^{\mathrm{b}}$ & $\uparrow$ & $13.1^{\mathrm{a}}$ & $14.4^{\mathrm{b}}$ & $15.2^{\mathrm{c}}$ & $\uparrow$ & $103,189,279$ \\
\hline Cheeses & $33.8^{\mathrm{a}}$ & $34.4^{\mathrm{a}}$ & $36.8^{\mathrm{b}}$ & $\uparrow$ & $14.5^{\mathrm{a}}$ & $15.1^{\mathrm{b}}$ & $16.7^{\mathrm{c}}$ & $\uparrow$ & $16,21,37$ \\
\hline Chocolate confectionary & $33.5^{\mathrm{a}}$ & $34.3^{\mathrm{a}}$ & $35.3^{\mathrm{b}}$ & $\uparrow$ & $14.2^{\mathrm{a}}$ & $14.8^{\mathrm{b}}$ & $15.7^{\mathrm{c}}$ & $\uparrow$ & $19,29,52$ \\
\hline Sugar based sweets & $33.7^{\mathrm{a}}$ & $32.4^{\mathrm{a}}$ & $30.2^{\mathrm{b}}$ & $\downarrow$ & $14.2^{\mathrm{a}}$ & $13.6^{\mathrm{a}}$ & $12.6^{\mathrm{b}}$ & $\downarrow$ & $10,25,52$ \\
\hline Fruit juice & 33.1 & 32.7 & 32.3 & & 13.8 & 13.8 & 13.4 & & $106,169,250$ \\
\hline
\end{tabular}

Larger portions of white and brown bread, boiled potatoes, breakfast cereals, fruit, vegetables and sugary sweets were associated with a reduction in total fat and saturated fat as $\%$ TE on the days in which the foods were consumed $(P \leq 0.05)$. An increase in total fat and saturated fat as \% TE was observed with increasing portions of eggs, milk, cheese and chocolate. Because these findings consider food portion size in relation to important markers of dietary quality, they may be useful in the development of specific food portion size guidelines aimed at Irish children into the future.

This project was funded by the Irish Government under the National Development Plan 2000-2006.

1. Food Standards Agency (2002) McCance and Widdowson's The Composition of Foods, 6th ed. Cambridge: Royal Society of Chemistry. 